BULL. AUSTRAL. MATH. SOC.

VOL. $21(1980), 479-480$.

\title{
THE MATHEMATICAL MODELLING OF CAPTURE-MARK-RECAPTURE EXPERIMENTS \\ ON ANIMAL POPULATIONS
}

\author{
Stuart F. Crosbie
}

In modelling a capture-mark-recapture experiment on an open animal population, one attempts to account for the predominant factors influencing its temporal structure. When too simple a model is used, parameter estimates tend to be biased and portray deceptively small sampling variances. On the other hand, adoption of an unnecessarily complex model causes a loss in precision. This thesis investigates the region of compromise between these two extremes.

A new general approach to capture-recapture modelling is proposed which, among other things, embodies all animals constituting part of the population at any stage of the experiment's duration as the principal modelling parameter, and places a probabilistic time of entry distribution on these individuals. By insisting that entry may only occur immediately before sampling occasions in conjunction with appropriate assumptions pertaining to survival and capture, one obtains a solution closely akin to that of the well-known Jolly-Seber model. Evidently, only those animals with length of residence happening to span a sampling time are accounted for. This deficiency is overcome if an animal's time of entry is assumed uniform between samples, thus rendering a modified Jolly-Seber solution.

Commencing with the latter solution, a series of models, possessing increasingly elementary structure, are considered by placing more restrictive assumptions on the key processes of birth, survival and

Received 17 March 1980. Thesis submitted to the University of Otago, November 1979. Degree approved: March 1980. Supervisor: Mr Bryan Manly. 
capture. These models appear algebraically intractable and so the likelihood must be optimized numerically. Through utilizing appropriate tests, one is able to select the simplest model providing an adequate fit to a given set of capture-mark-recapture data. A comprehensive FORTRAN IV computer program, CMR, has been written to perform this model selection process.

In concurrence with Jolly's developments (to be published shortly), the models in this thesis represent a reversal against recent trends to generalize the Jolly-Seber model. The population studies analysed by program CMR thus far, have all been found to provide empirical justification in favour of such a move. This in no way discredits the more complex models but, rather, reinforces the need for the biologist to have a wide range of models from which to choose. We have endeavoured to fulfil that need whenever the Jolly-Seber model is likely to be more complex than required. 